\title{
Preventive Intervention
}

National Cancer Institute

\section{Source}

National Cancer Institute. Preventive Intervention. NCI Thesaurus. Code C15843.

Therapeutic, nutritional, environmental, social and/or behavioral interventions at the societal, community, organizational or individual levels to reduce, modify or stop the course of a disease. 